\title{
Artefacts in the deconvolution of images of E-S0 galaxies
}

\author{
R. Michard \\ 1 Observatoire de la Côte d'Azur, Dept. Augustin Fresnel, B.P. 229, F-06304 Nice Cedex 4, France \\ 2 Observatoire de Paris, DEMIRM, 77 av. Denfert-rochereau, F-75014 Paris, France
}

Received August 11; accepted November 27, 1995

\begin{abstract}
Unexpected circumnuclear isophotal structures were observed during tests of Lucy's deconvolution technique, performed upon high resolution CFHT frames. They contain an enhanced nuclear disk surrounded by a "boxy" ring; an upward hump in ellipticity corresponds with this ring. Such structures are produced in the deconvolution of synthetic images having large enough surface brightness gradients and ellipticities, as is the case for two of our test galaxies. Similar stuctures occur in convolving and deconvolving model galaxies with a model of the HST's PSF prior to the telescope renovation, but they are limited in radial extent up to less than 1 arcsec of isophotal major axis. If the deconvolution is performed with a PSF differing somewhat from the one used in the convolution, large and far extending artefacts can occur.
\end{abstract}

Key words: galaxies: elliptical and lenticulars, $\mathrm{cD}$ — galaxies: photometry — techniques: image processing

\section{Introduction}

As part of an ongoing survey of color distributions in E-S0 galaxies, we are studying high resolution $B$ and $R$ frames obtained with the CFHT by J.-L. Nieto in 1989-91 and by P. Poulain in 1992. It was deemed useful to test the application of Lucy's deconvolution technique to such good frames, whenever the PSF could be accurately measured from a suitable star.

Tests were made on frames of the three Leo group objects NGC 3377, 3379 and 3384. The red images have PSF's in the range of $0.5-0.6$ arcsec FWHM, the blue ones being broader by some $0.2 \operatorname{arcsec}$. For two of these, i.e. 3377 and 3384 , we found, after deconvolution, exciting isophotal structures surrounding the nucleus: these include an innermost disky region of about 1.5 arcsec major axis, surrounded by a narrow boxy ring. Farther out, starts the "normal" disky range easily measured from the original frames.

Similar structures have already been described in a series of papers discussing HST frames of a sample of galaxies, mostly elliptical, in the Virgo cluster, by Jaffe et al. (1994), van den Bosch et al. (1994), Ferrarese et al. (1994). They have termed "type II" objects, such galaxies having a nucleus much sharper than the isothermal model, and showing the inner circumnuclear disk. Most of these, i.e. NGC $4342,4550,4564,4570,4623$ are more typical of lenticulars than of ellipticals (see Michard \& Marchal 1994, for a so-called "quantitative morphology"

Send offprint requests to: R. Michard of 103 RSA E-S0 galaxies). For NGC 4564, 4570, and 4623, the structures are alike those found in our tests, while they may be more complicated in other cases.

It was thought necessary to check the significance of the detected structures by deconvolving model galaxies. This has indeed been done by van den Bosch et al., but they explicitly mention only one light distribution, a modified Hubble model given in Eq. (3) of their paper. This distribution is broad, with an FWHM of 1.82 arcsec, and rather flat topped. It would be largely resolved in our good CFHT frames, as is for instance the core of NGC 3379. The nucleus of the so-called "type II" galaxies, or of NGC 3377 and 3384 that showed "exotic" structures, is much sharper and largely unresolved: see Ferrarese et al. (1994) for the nuclear profiles of the HST sample after deconvolution.

The present note summarizes our experiments with model galaxies, completely devoid of exotic structures before being convolved and deconvolved by the PSF. It is found that models having a nucleus of sharp light gradient (surrounded by a region of lesser slope) and a not too small ellipticity, acquire novel structures in the deconvolution operation. The importance of "isophotal anomalies" increases with the contrast of the nucleus against the surroundings; it also increases, at first, with the number of iterations in Lucy's technique, then seems to stabilize. The FWHM of the PSF and the importance of its wings, influence the amplitude and radial extent of these anomalies.

Following the advice of Dr. Lucy, who acted as referee for a preliminary version of this paper, an examination was made of artefacts induced by convolving and deconvolving 
sharply peaked model galaxies, with a model of the Hubble Space Telescope PSF before the spherical aberration of the primary was compensated for. Since the actual PSF, for a given HST frame, is generally not observed, we also made experiments upon the effects of differences in the convolving and deconvolving PSF's.

Remark: The numerical experiments described below could perhaps be completed, or partly replaced, by an analytical approach. Simple analytical models are indeed sufficient to display all kinds of artefacts observed with more complex model galaxies. This interesting approach is, however, outside the scope of this incidental contribution.

\section{Experiments with model galaxies and "seeing- like" PSF's}

Throughout the present experiments, we used the MIDAS software to build model galaxies, to convolve these with the adopted PSF's, and most notably to deconvolve the resulting frames with Lucy's technique.

We deal first with "seeing-like" PSF's, meaning they can be roughly approximated by gaussian functions, eventually completed by moderately extended wings. In this case, the deconvolutions were generally limited to 18 iterations, although an experiment was made of pushing the iterations to 99 steps, with no noticeable change in the results, as far as the geometry of isophotes are concerned. No artificial noise was introduced, as our purpose is to detect deconvolution artefacts, not to estimate the accuracy of results derived from noisy data. A model passed through the operations of convolution plus deconvolution with the PSF will be said to be processed.

The PSF's were generally gaussian with $\sigma_{*}$ values of 2 , 3 or 4 pixels. Assuming the pixel to be 0.1 arcsec, the conditions are quite similar to the ones encountered with the CFHT camera (pixel 0.107 arcsec). We have also experimented with a PSF having enhanced wings as compared to a pure gaussian, and also with a measured PSF from one of our CFHT frames.

\subsection{Exponential disks with a gaussian nucleus}

A number of experiments were performed using pure exponential disks with elliptical isophotes (with various ellipticities $\epsilon$ ), and with scale parameters in the range of 10-20 times $\sigma_{*}$. Eventually, a central gaussian nucleus of equal $\epsilon$ was added to the disk. Tests were made with several values of the contrast $C$ of the nucleus against the disk, which was varied between $C=0$ (pure disk) and $C=10$ (peak nucleus brightness equal to 10 times the peak disk brightness). The equivalent $\sigma_{\mathrm{r}}$ of the nucleus, that is the quantity $\left(\sigma_{x} . \sigma_{y}\right)^{1 / 2}$ was also varied, from well below to well above $\sigma_{*}$. Note that the axis ratio of the nucleus is $\sigma_{y} / \sigma_{x}$. Some results will be summarized here:
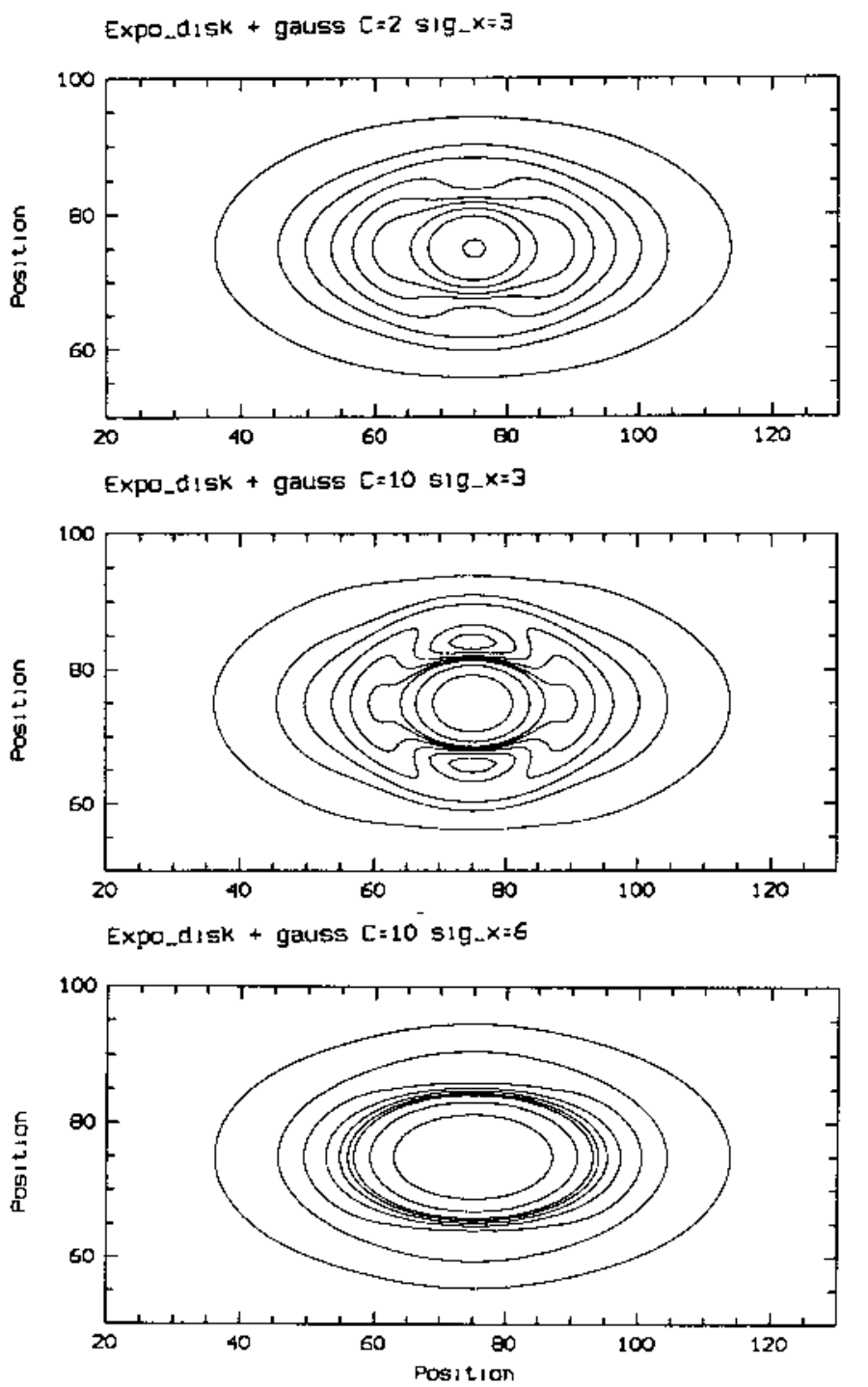

Fig. 1. Isophotes of "processed" frames of models of constant $\epsilon=0.5$, formed of an exponential disk and a gaussian nucleus. This is defined by its contrast $C$ against the disk, and the $\sigma_{x}$ value (here $\sigma_{y}=\sigma_{x} / 2$ ). The PSF is the gaussian with $\sigma_{*}=3$ pixels. From top to bottom: $C=2$ and $\sigma_{x}=3$ : narrow nucleus of small contrast; $C=10$ and same $\sigma_{x}$ : narrow nucleus of strong contrast; $C=10$ and $\sigma_{x}=6$ : broad nucleus of strong contrast

1. For $\epsilon=0$ the processed frame always shows a system of dark and bright rings surrounding the central peak, whatever the value of $C$. This system is of very low contrast for the pure disk; in this case, the deconvolution restores the input frame with errors of less than $1 \%$, except at the central maximum, where it reaches $3 \%$. Such oscillations about the correct solution are known as the Gibbs's phenomenon, or sometimes as "ringing". 
(a) $R *(1 / 4)$ lau $R_{-} e=80 \quad c / a=0.5$ Gauss 5 Ig=2

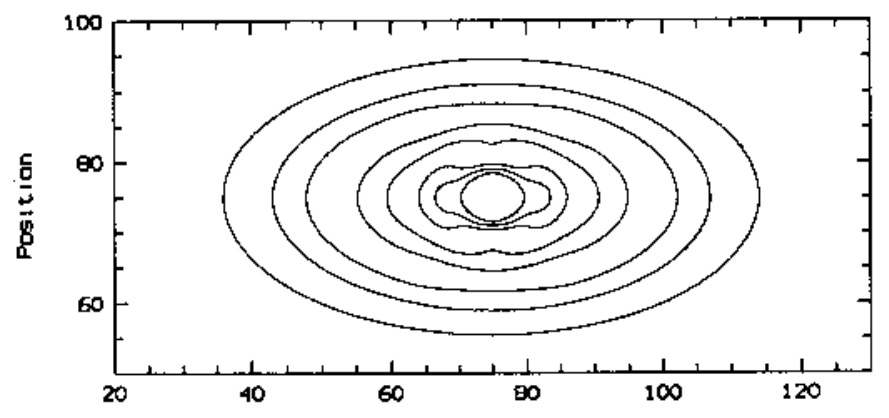

(b) R*o(1/4) lau R_e=80 c/a=0.5 Gauss sig=3
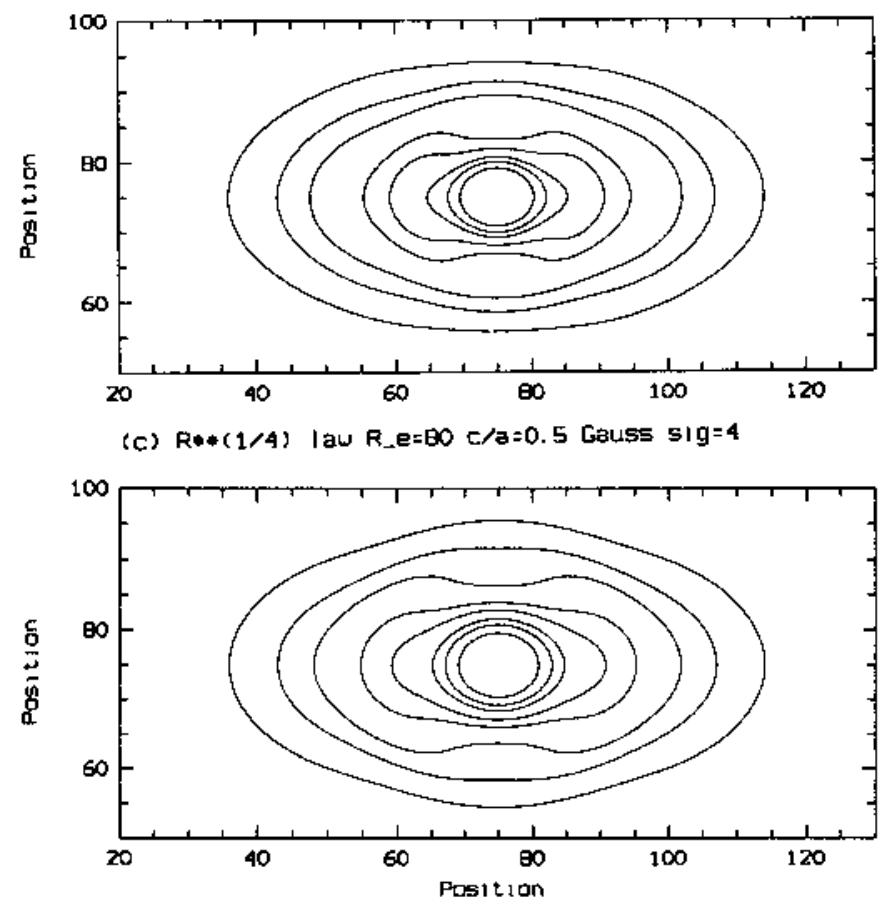

Fig. 2. Isophotes of "processed" frames from an $r^{1 / 4}$ model with an equivalent de Vaucouleurs radius $r_{e}=80$ pixels and $\epsilon=.5$. Three different PSF's with $\sigma_{*}$ values of 2,3 , 4 were used

2. When the sharp gaussian nucleus is introduced, still with $\epsilon=0$, the system of rings shows a strongly increasing contrast. The geometry of the isophotes remains of course unaffected.

3. When $\epsilon$ is significantly larger than zero, the system of rings is still traceable, but with strong differences between the major and minor axis, so that large errors in the geometry of the restored frame are introduced: these can be of the modest kind met in our observations, with the inner disk and boxy ring; they can be much more spectacular, with detached crescents in the isophotal maps, the darker crescents normal to the minor axis being the most "robusts" of these artefacts.
Figure 1 illustrates these effects with the isophotal contours of processed frames for three models of ellipticity $\epsilon=0.5$, processed with the gaussian PSF having $\sigma_{*}=3$ pixels. Two of these have a sharp nucleus, with $\sigma_{x}=3$, and two different contrasts, i.e. 2 and 10. The lower contrast nucleus leads to simple isophotal distorsions, with inner disk and boxy ring, while the other results in a curious pattern, with intense dark crescents normal to the minor axis. These tend to disappear when the nucleus is broadened, as shown by the third model with $\sigma_{x}=6$.

Remark: If the parameter $\sigma_{x}$ is lessened (or equivalently the $\sigma_{*}$ of the PSF) the geometry of the isophotes remains qualitatively similar to the case $\sigma_{x}=\sigma_{*}=3$ shown in Fig. 1, but the radial scale of the perturbation is lessened.

\section{2. de Vaucouleurs $r^{1 / 4}$ law}

A number of $r^{1 / 4}$ models have been considered, with radius $r_{e}$ in the range $10-40 \sigma_{*}$, elliptical isophotes and various ellipticities. Such models have very strong central gradients. In the MIDAS implementation of calculating $r^{1 / 4}$ models, the central pixel has the maximum $\mathrm{SuBr}$ (Surface Brightness) of de Vaucouleurs law, 8.34 magnitudes above the $\mathrm{SuBr}$ at $r_{\mathrm{e}}$. This extremely bright central pixel is perhaps unrealistic, so that we also experimented modified $r^{1 / 4}$ models: in this case the $r^{1 / 4}$ component is filtered with a gaussian, much narrower than the adopted PSF, but broad enough to smooth out the central pixel. The filter can be either circular of elliptical. It should be noted that this operation conserves the flux of the innermost pixels, so that the thus modified $r^{1 / 4}$ core keeps a strong contrast against the surroundings. The tests showed that pure or modified such models lead to closely alike deconvolved images.

Figure 2 illustrates the variations in the geometry of the deconvolved images, from a unique $r^{1 / 4}$ model, but with 3 different gaussian PSF's having $\sigma_{*}$ values of 2,3 and 4 pixels respectively. The equivalent de Vaucouleurs radius $r_{\mathrm{e}}=(a c)^{1 / 2}$ of the model was 80 pixels and its ellipticity 0.5 . The "isophotal anomalies" have clearly the same characters with the three PSF's, but with a variation of radial extent: with a broader PSF, they extend farther from the nucleus.

Figure 3 gives an exemple of artefacts introduced in the processing of modified $r^{1 / 4}$ models of various ellipticities, with $\epsilon$ values of $0.3,0.5$ and 0.6 respectively. These models have the same de Vaucouleurs equivalent radius $r_{\mathrm{e}}$, with $r_{\mathrm{e}}=(a c)^{1 / 2}=50$ pixels; the PSF was the gaussian with $\sigma_{*}=3$ pixels. In this case it was chosen to show the essential isophotal parameters, that is $\epsilon$ and $e_{4}$, against the equivalent radius $r$ of the contours. In our applications of Carter's isophotal analysis, $e_{4}$ is the coefficient of $\cos 4 \omega$. Clearly, the two parameters show correlated variations for the three models, with minima and maxima at common abscissae. The $\epsilon$-profiles depart strongly from 


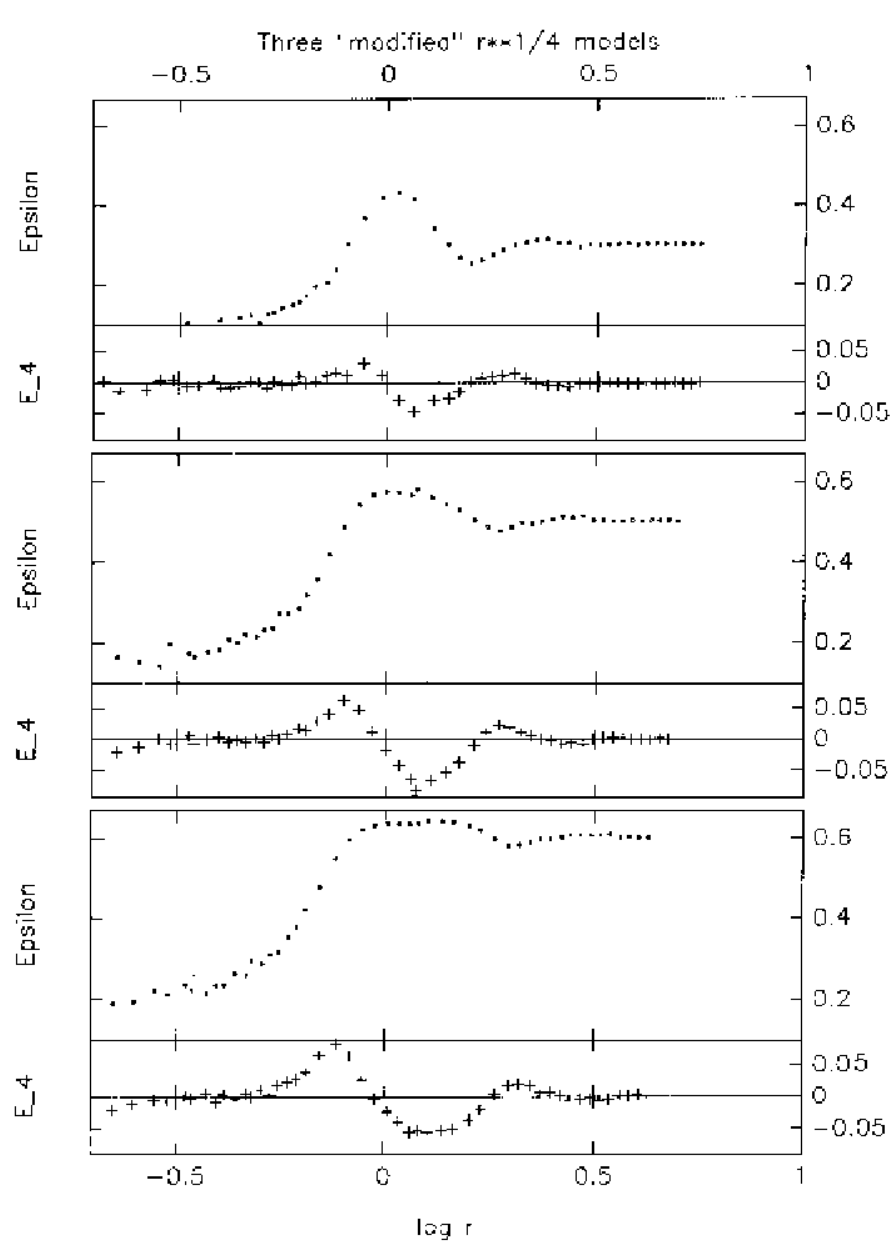

Fig. 3. Partial results of processed frames from modified $r^{1 / 4}$ models of ellipticities $0.3,0.5$ and 0.6 . These models have the same equivalent de Vaucouleurs radius $r_{\mathrm{e}}=50$ pixels. while the PSF is the gaussian with $\sigma_{*}=3$. The graphs show the $\epsilon$ and $e_{4}$ parameters, $e_{4}$ being the coefficient of $\cos 4 \omega$ in Carter's isophotal representation. The abscissae are $\log _{10} r$, with $r=(a c)^{1 / 2}$ being the equivalent radius of the contours, in the unit of 10 pixels (or 1 arsec to relate this experiment with realistic observational circumstances). Note that the true ellipticities are not restored, while $e_{4}$ shows a characteristic variation around its true zero value

the expected constants: they show a kind of damped oscillations about the correct values. This is also true for the $e_{4}$-profile, with an inner disky region surrounded by a boxy ring (compare with the central graph of Fig. 2, which gives the isophotal contours for a similar model). The perturbations in geometrical parameters extend to about 5 times the FWHM of the PSF.

\subsection{Mixed models involving a bulge and an exponential disk}

The bulge could be either a single $r^{1 / 4}$ component, or the sum of two such components of different de Vaucouleurs

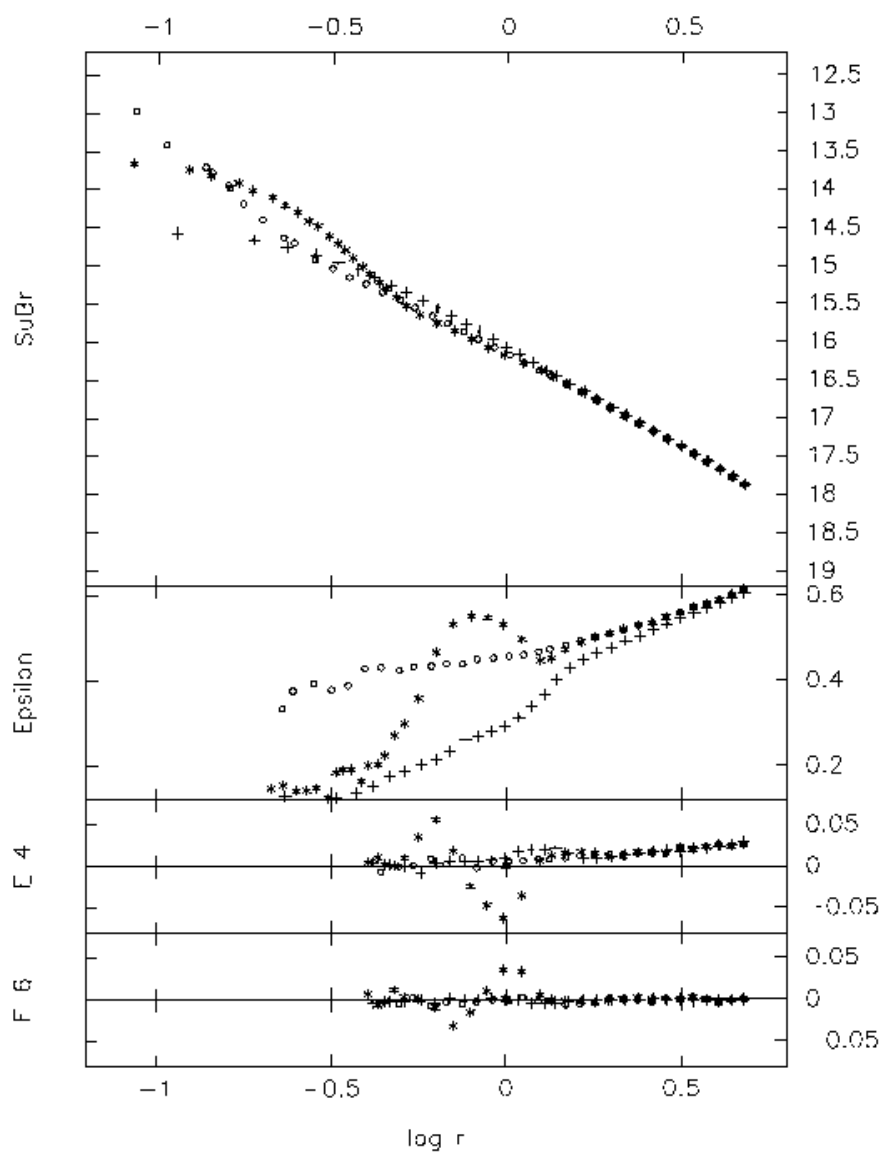

Fig. 4. Comparison of a model akin to NGC 4570 with its PSF convolved image, and with the deconvolved one. The PSF is an observed one from a CFHT frame of 0.59 arcsec FWHM. The abscissae scale is the mean radius $r$ in arcsec and logarithms. The circles refer to the model, the crosses to the convolved image, the stars to the deconvolved one with 40 iterations. The graphs are truncated to the radial range were the parameters are not much affected by unsufficient sampling. Upper graph: Mean isophotal $\mathrm{SuBr}$, in magnitudes per sq. arcsec. Intermediate graph: Ellipticity. Lower graphs: The $e_{4}$ and $e_{6}$ coefficients

radii and ellipticities: such composite bulges are intended to give a better approximation to the actual bulges of S0 galaxies. Recent analysis (Scorza \& Bender 1990, 1995; Simien \& Michard 1990) indicate that the bulges do not have a constant ellipticity, but rather that $\epsilon$ increases from its central value to some maximum, then either remains constant or increases again. Michard (1994) gives statistics of this property.

Mixed models lead to similar anomalies in the internal isophotes as found for the simple $r^{1 / 4}$ models. Figure 4 gives results for one such model, which was intended to be analogous to NGC 4570, according to various litterature data: Ferrarese et al. for the run of the $\mathrm{SuBr}$, Michard \& Marchal (1993) for the disk axis ratio. In this case the PSF was taken from a CFHT frame with an FWHM of 
0.59 arcsec. Figure 4 compares the runs of the $\mathrm{SuBr}$, the ellipticity, the $e_{4}$ and $e_{6}$ coefficients for the model used here, and its convolution and deconvolution (after 40 iterations). Obviously the deconvolution is not able to restore the sharp central peak of the $r^{1 / 4}$ law, although smoothed as indicated in 2.2. The errors in the $\epsilon$ and $e_{4}$ parameters of the deconvolved image, are very similar to those shown in the above experiments (see Fig. 3). The $e_{6}$ coefficient shows similar oscillations as $e_{4}$. Note a range of spurious diskyness in the convolved image at a radius somewhat above 1 arcsec.

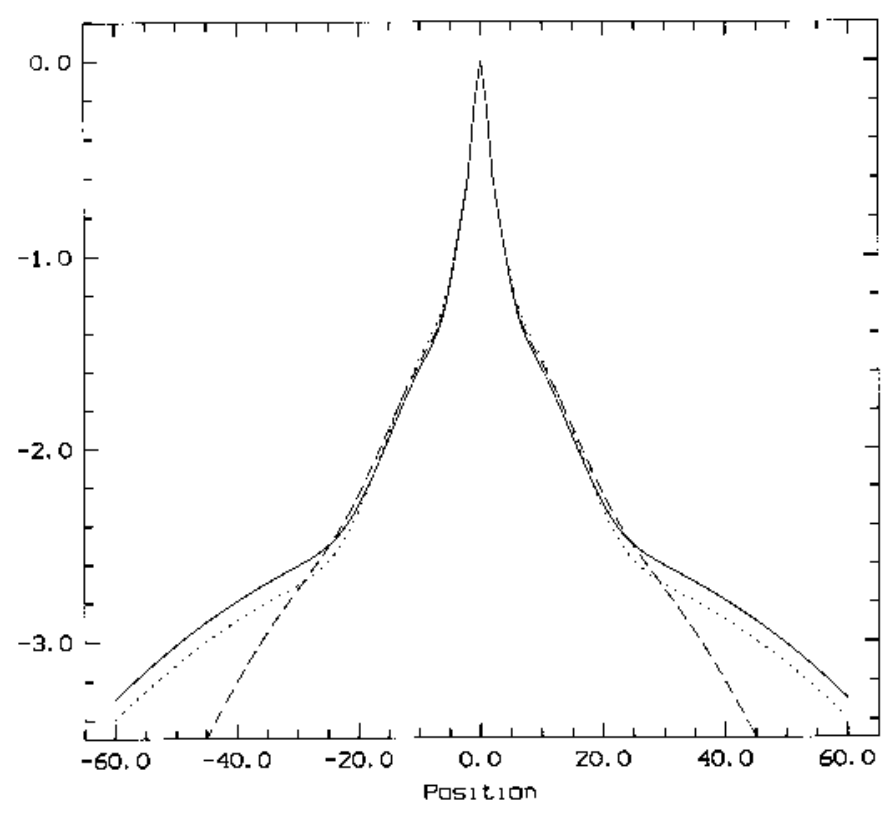

Fig. 5. Radial profiles of model PSF's in a logarithmic scale. Abscissae: pixels of 0.043 arcsec. Solid line: phst0. Dots: phstx. Dashs: phsty. These have been used to study the effects of wing extent

\section{Experiments with "HST-like" PSF's}

The following experiments might be relevant to the discussion of HST results about the nuclear structure of earlytype galaxies, if the data were obtained before the compensation of the main mirror aberration. Prior to this operation, the HST PSF contained a sharp core of about 0.1 arcsec FWHM, surrounded by a very broad halo (Burrows et al. 1991). The PSF was dependant upon the camera, the position in the field, and also upon time. The instrument users were often not able to observe the PSF relevant to a given frame, but could rely upon an available library of PSF's, or more generally upon software provided to generate model PSF's (Hodge et al. 1993; Krist 1993). Mo \& Hannisch (1993), compare an observed PSF, a relevant library PSF and a model PSF obtained from the Tiny Tim

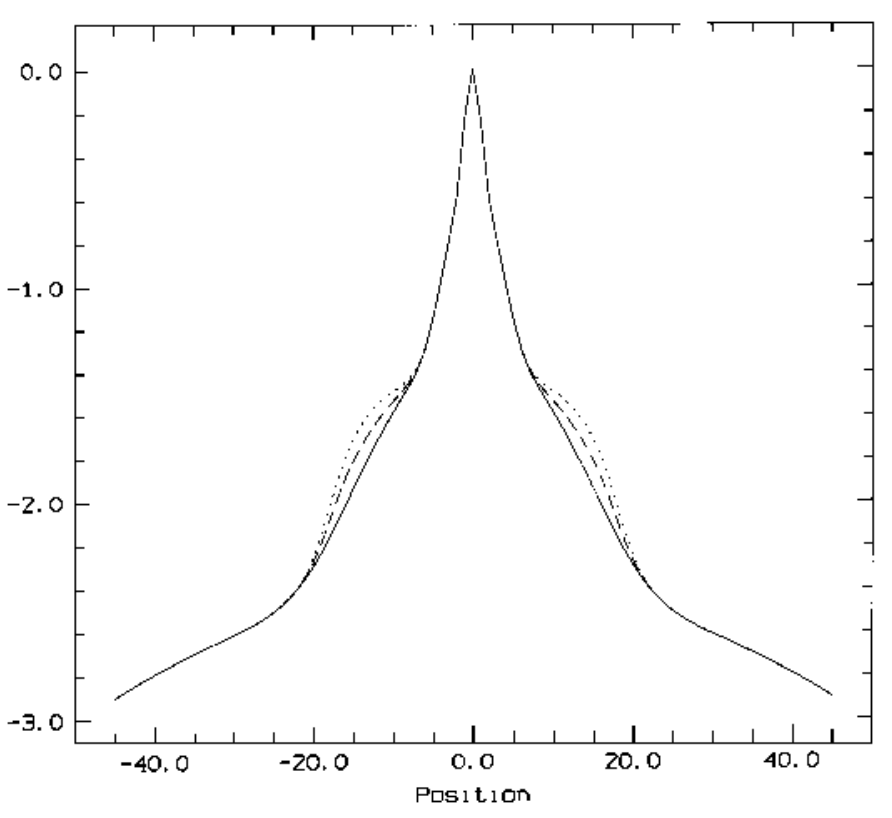

Fig. 6. Radial profiles of model PSF's in a logarithmic scale. Abscissae: pixels of 0.043 arcsec. Solid line: phst1. Dots: phst1p. Dashs: phst $1 m$. These have been used to study the effects of plateau level

software. There are quite surprising differences between the three functions, notably in their wing extent.

For the present experiments, we used several PSF's with circular symmetry, built with a pixel size of 0.043 arcsec, the one of the Planetary Camera (and rather undersampling the central peak).

1. the PSF phsto was built from a series of 4 gaussians, and is intended to approximate the mean radial profile of the HST PSF as given by Burrows et al. (1991). It was truncated at a radius of 60 pixels or 2.58 arcsec.

2. the PSF's phst10, phst 1 and phst2 are the same as phst0 but truncated to radii of 52,45 and 30 pixels respectively (that is 2.24, 1.94 and 1.29 arcsec).

3. for the PSF phstx and phsty the last terms of the series were modified to get less extended wings, more alike the Tiny Tim model quoted by Mo and Hannisch. $p h$ sty was truncated to a radius of 50 pixels or 2.15 arcsec.

4. since the HST PSF's have a remarkable plateau at radii around 0.7 arcsec, the PSF's phst $1 p$ and phst $1 m$ are intended to study, together with phst1, the effects of errors in the level of this feature in the assumed PSF. The feature is small in phst1, strong in phst1p, while phst $1 m$ is a mean of the other two.

5. the PSF phstj2 is the same as phst2 but broadened by a jitter of some 0.02 arcsec RMS; the FWHM is increased by $10 \%$.

6. the PSF phstn is an approximation to the "new" HST after repair. 
Figure 5 gives the radial profiles of phsto, phstx and phsty. Figure 6 compares the radial profiles of the PSF's phst1, phst $1 m$ and phst1p.

\subsection{Experiments with a unique PSF and various models}

To save computing time these experiments were made with phst2. It will appear that the results are not significantly changed with other HST-like PSF's, provided that the same PSF is used at convolution and deconvolution. A series of models were built by adding the following components:

1. a unique bulge of axis ratio 0.5 , following the $r^{1 / 4}$ law, with a mean de Vaucouleurs radius $r_{\mathrm{e}}=8$ arcsec. The central pixel was attenuated by a mild gaussian filtering. The $\mathrm{SuBr}$ at the effective radius was unity.

2. four different gaussian nuclei $n u_{i j}$ were the first index refers to the width of the feature, the second to its peak $\mathrm{SuBr}$. If $i=1$ the FWHM of the nucleus is equal to the one of the HST PSF, i.e. 0.1 arcsec. If $i=2$ it is twice as large. For $j=1$ the peak $\mathrm{SuBr}$ of the nucleus is 1000 in the unit specified above, 1 magnitude larger for $j=2$.

3. two exponential disks $d 1$ and $d 2$ the first having the same axis ratio of 0.5 as the bulge, the other being much more inclined, with an axis ratio of 0.3 .

Table 1 summarizes the results of these experiments, using a number of ad hoc parameters to describe the measured deviations of the image deconvolved with 80 iterations from the corresponding input model. Figure 7, giving the $\mathrm{SuBr}, \epsilon, e_{4}$ and $e_{6}$ profiles for 3 different stages of deconvolution, is also of interest. The following comments may be made:

1. the effects of convolving the models with any HSTlike PSF, including the truncated phst2, are rather terrific upon the peak $\mathrm{SuBr}$ and the ellipticity profiles. It also introduces a spurious diskyness in a limited radial range.

2. the deconvolution is rather successful in restoring the $\mathrm{SuBr}$ profiles, but leaves errors in the ellipticity curves, which show oscillations about the nominal values. Errors at the 0.025 level in $\epsilon$ may extend up to 1 arcsec (major axis!), while errors of 0.01 may extend farther than 2 arcsec for models with the "broad" nucleus.

3 . artefacts of the type inner disk + boxy ring are regularly produced, at various "contrasts". The contrast of the boxy ring, measured from the minimum value of $e_{4}$, is maximum when the sharp nucleus is both narrow and bright, as expected from our preliminary experiments with gaussian PSF's. For the nucleus nu22, the one containing the largest energy, the oscillations in the $e_{4}$ profile lead to two boxy rings, and detected errors in the restored $e_{4}$ extend up to 3 arcsec.

4. the introduction of a disk in the model, that is of a lower gradient region around the bulge, does not change significantly the amplitudes or radial extents of errors in the restored isophotes.

5. pushing farther and farther the iterations, certainly improves the restoration of the $\mathrm{SuBr}$ curve, but it is not so for the geometrical parameters. It may be seen from Fig. 7, that the errors in the $\epsilon$-profile, and the $e_{4}$ and $e_{6}$ coefficients are enlarged, although restricted to a smaller radial range.

\subsection{Experiments with a unique model and various PSF's}

Using a particular model of the above series, experiments were made of convolving and deconvolving with a number of PSF's. The results are summarized in Table 2, giving the same information as Table 1, in the same format. The first few lines of this table refer to the case where the same PSF is used for both blurring and deconvolving. Then the results do not differ much from those obtained with the truncated PSF (repeated in Table 2 for easier reference): the successes and artefacts in the restoration are approximately the same for the same number of iterations.

Of special interest are the results of processing the test model with the phstn PSF, the one without spherical aberration. The "ringing" of the solution leaves artefacts qualitatively similar to those encountered with PSF's adequate to the "old" HST, but their geometrical scale is much smaller. With 80 iterations, the success of Lucy's deconvolution at restoring the correct $\mathrm{SuBr}$ profile of the model is almost perfect!

To simulate the uncertainties in the choice of the deconvolving PSF, experiments where also made with different PSF's at convolution and deconvolution. When using a PSF slightly broadened by jitter at convolution, the deconvolution was almost as successful as with identical PSF's. However the situation was quite different when deconvolving with a PSF having less extended wing than the convolving one, or with a different "plateau".

When the deconvolving PSF is abruptly truncated, (PSF's phst1 and phst10), the results are unchanged for the isophotes inside $r<1$ arcsec, but new artefacts occur in the $\epsilon$-profile and other geometrical parameters at large $r$ values. A reduction of the wing extent of the assumed PSF by a smooth correction, as with phstx and phsty, also leads to errors extending at very large radial distances from the galaxian center (see Fig. 8).

On the other hand, the change in the level of the plateau leads to errors in a more restricted radial range, with large artefacts at $r<1$ and smaller ones at $r<2$ arcse (see Fig. 9).

\section{Discussion and conclusion}

It has been shown that the inner isophotes of oval model galaxies containing a sharp central feature are more or less distorted in the process of convolution by the PSF, 
Table 1. Deconvolution experiments with various models and the PSF phst2. (1) Model components. (2) Peak model SuBr in magnitudes (arbitrary scale). (3) Used PSF for convolving. (4) Used PSF for deconvolving. (5) Peak magnitude difference after convolution. (6) Peak magnitude difference after deconvolution. (7) Major axis range where the $\epsilon$ error is 0.025 or more (arccec). (8) Major axis range where the $\epsilon$ error may reach 0.01. (9) Major axis range of erroneous $e_{4}$. (10) Minimum $e_{4}$ in a boxy ring, in percent. (11) Position of this minimum

\begin{tabular}{lllllllllll}
\hline$(1)$ & $(2)$ & $(3)$ & $(4)$ & $(5)$ & $(6)$ & $(7)$ & $(8)$ & $(9)$ & $(10)$ & $(11)$ \\
\hline bul2 & 12.17 & phst2 & id & 2.97 & 1.38 & 0.58 & 0.93 & 0.95 & -2.2 & 0.53 \\
bulg & 13.24 & phst2 & id & 2.06 & 0.64 & 0.58 & 0.93 & 0.95 & -1.9 & 0.56 \\
nu11+bulg & 12.36 & phst2 & id & 2.38 & 0.50 & 0.69 & 1.68 & 0.85 & -4.0 & 0.63 \\
nu12+bulg & 11.70 & phst2 & id & 2.53 & 0.41 & 0.74 & 1.68 & 0.85 & -6.7 & 0.64 \\
nu21+bulg & 12.36 & phst2 & id & 1.46 & 0.23 & 0.80 & 2.80 & 0.97 & -3.8 & 0.69 \\
nu22+bulg & 11.70 & phst2 & id & 1.12 & 0.11 & 0.92 & 2.81 & 2.90 & -3.5 & 0.76 \\
nu12+bulg+d1 & 11.70 & phst2 & id & 2.47 & 0.44 & 0.72 & 1.44 & 1.00 & -7.2 & 0.63 \\
nu12+bulg+d2 & 11.70 & phst2 & id & 2.47 & 0.44 & 0.76 & 0.81 & 1.05 & -6.7 & 0.64 \\
nu22+bulg+d1 & 11.70 & phst2 & id & 1.90 & 0.11 & 0.89 & 1.44 & 2.90 & -5.4 & 0.82 \\
nu22+bulg+d2 & 11.70 & phst2 & id & 1.90 & 0.11 & 0.88 & 1.05 & 3.80 & -5.5 & 0.83 \\
\hline
\end{tabular}

Table 2. Deconvolution experiments with one model and various PSF's. The disposition is the same as for Table 1

\begin{tabular}{lllllllllll}
\hline$(1)$ & $(2)$ & $(3)$ & $(4)$ & $(5)$ & $(6)$ & $(7)$ & $(8)$ & $(9)$ & $(10)$ & $(11)$ \\
\hline nu12+bulg & 11.70 & phstn & phstn & 1.20 & 0.02 & 0.35 & 1.06 & 1.01 & -5.6 & 0.33 \\
nu12+bulg & 11.70 & phst2 & phst2 & 2.53 & 0.41 & 0.74 & 1.68 & 0.85 & -6.7 & 0.64 \\
nu12+bulg & 11.70 & phst0 & phst0 & 2.78 & 0.50 & 0.78 & 1.69 & 1.25 & -6.1 & 0.64 \\
nu12+bulg & 11.70 & phsty & phsty & 2.66 & 0.42 & 0.75 & 1.69 & 0.78 & -6.2 & 0.64 \\
nu12+bulg & 11.70 & phstj2 & phst2 & 2.60 & 0.54 & 0.74 & 1.68 & 2.60 & -6.6 & 0.63 \\
nu12+bulg & 11.70 & phst0 & phsty & 2.78 & 0.57 & 4.94 & 7.77 & 5.90 & -5.7 & 0.66 \\
nu12+bulg & 11.70 & phst0 & phstx & 2.78 & 0.51 & 4.10 & 4.80 & 5.30 & -6.8 & 0.82 \\
nu12+bulg & 11.70 & phst0 & phst1 & 2.78 & 0.56 & 4.68 & 5.10 & 4.90 & -6.7 & 0.65 \\
nu12+bulg & 11.70 & phst0 & phst10 & 2.78 & 0.53 & 4.62 & 4.95 & 4.95 & -5.2 & 0.67 \\
nu12+bulg & 11.70 & phst1m & phst1 & 2.75 & 0.54 & 2.00 & 3.25 & 4.20 & -12.0 & 0.75 \\
nu12+bulg & 11.70 & phst1m & phst1p & 2.75 & 0.45 & 1.90 & 2.25 & 2.80 & -7.4 & 1.39 \\
\hline
\end{tabular}

followed by deconvolution. In this context, we might perhaps offer an operational definition of a "sharp central feature": it is anything with an FWHM smaller, or not much larger, than the FWHM of the PSF. It thus can be an $r^{1 / 4}$ bulge, with a theoretical FWHM of $1.33410^{-4} r_{\mathrm{e}}$; or one of the modified $r^{1 / 4}$ laws introduced here, with less extreme FWHM's; or simply a gaussian nucleus not fully resolved by the PSF.

In such situations, it is found that the ellipticity profile of the model is not correctly restored, but develop a kind of damped oscillation about the input $\epsilon$-curve. At the same time, a small inner disky region, surrounded by a boxy ring, appears in the processed object, just as was found in our tests upon CFHT frames of NGC 3377 and 3384. Multiple oscillations in the geometrical parameters can sometimes be observed, similar to the "ringing" of the restored $\mathrm{SuBr}$ profile.

While the deconvolution by Lucy's technique is effective in approaching the true light profile of a sharply peaked galaxy, it is unsuitable to recover the correct geometrical properties of the core, notably the ellipticity. Dr. Lucy proposes that his new technique of "two channels deconvolution" (Lucy 1993; Hook \& Lucy 1993) might be useful for this purpose. To quote him: "The result would be a statement that a certain percentage of the galaxy's light originate in an unresolved nucleus, which the available data does not allow one to distinguish from a point source." Since this technique eliminates the Gibbs oscillations, it would possibly also lead to a correct restoration of the geometry of the core.

As regards the findings of van den Bosch et al about their "type II" galaxies, one can wonder about the limit of significance of their peculiar isophotal geometry. van den Bosch has commented in a private communication, that the artefacts here described do not extend farther than 5 times the FWHM of the PSF, according to his own tests, making reliable the published HST results farther than 0.5 arcsec.

Our own experiments above are not in gross disagreement with this opinion: provided that the actual HST PSF was exactly known and untruncated, and also that criteria for accepting geometrical parameters are not too severe, the 0.5 arcsec limit will not be greatly exceeded. On the other hand, small differences between the assumed PSF 


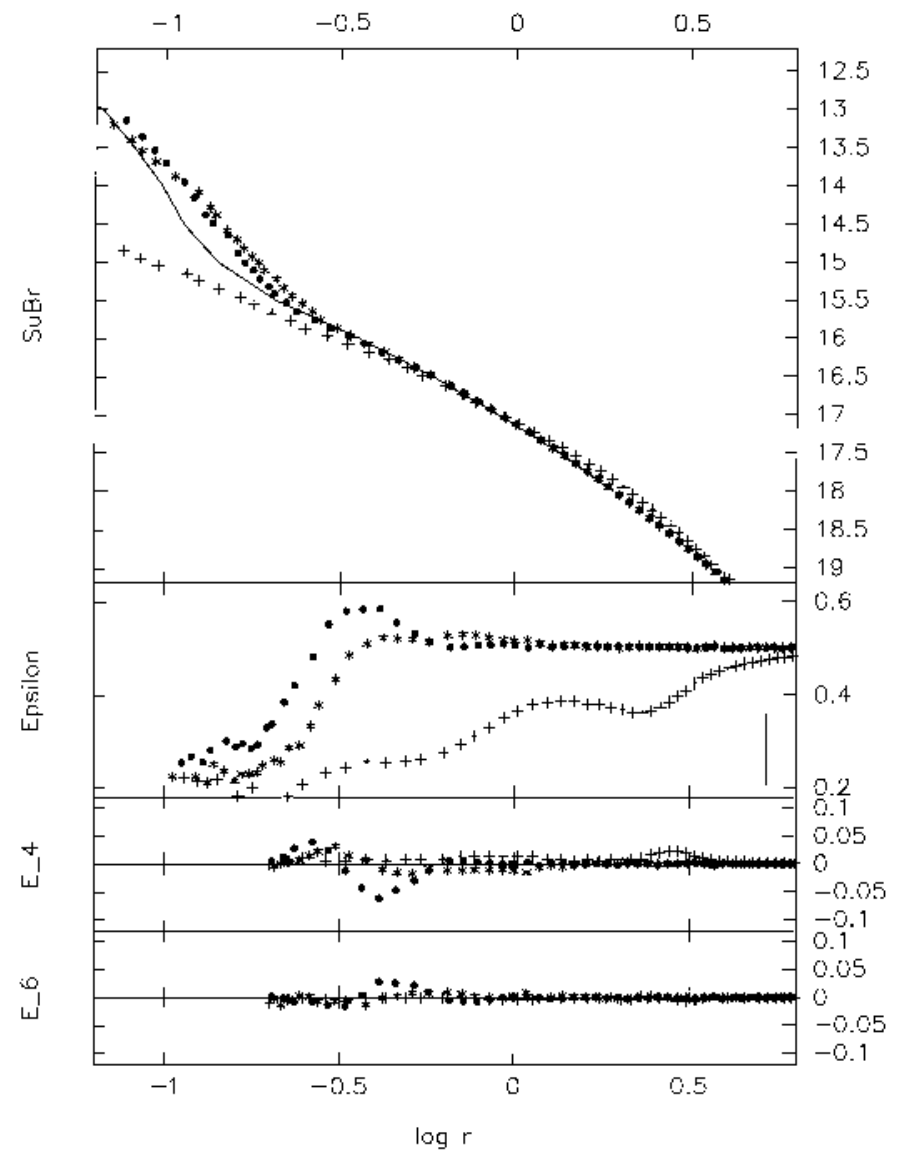

Fig. 7. Results of convolution and deconvolution of a bulge + nucleus model by the PSF phst0. The model has a constant $\epsilon=0.5$ and elliptical isophotes. The abscissae scale is the mean radius $r$ in arcsec and logarithms. Solid line: model $\mathrm{SuBr}$. The crosses refer to the convolved image, the stars to the deconvolved one with 40 , and the dots with 80 iterations. The graphs are truncated to the radial range were the parameters are not much affected by unsufficient sampling. Upper graph: Mean isophotal $\mathrm{SuBr}$, in magnitudes per sq. arcsec. Intermediate graph: Ellipticity. Lower graphs: The $e_{4}$ and $e_{6}$ coefficients

and the one actually realised by the instrument for a specific frame, may lead to large and far extending errors in the isophotal geometry of restored galaxian images. It may be argued that the errors found above when deconvolving with notably truncated PSF's, or PSF's with attenuated far wings, are too large to be mistaken for galaxian properties! The peculiar $\epsilon$-profiles would, if real, appear in ground based data. The case is less simple for errors shown in the experiment where the plateau of the PSF was modified. With more subtle differences than introduced here, between the convolving and deconvolving PSF's, a large variety of artefacts could probably be produced.

van den Bosch also insists that "nuclear disks" are to be seen in the original HST frames of the S0 galaxies NGC 4342,4570 and 4623 . There can be of course little doubt that the disk of S0's, and also of disky E's, may extend

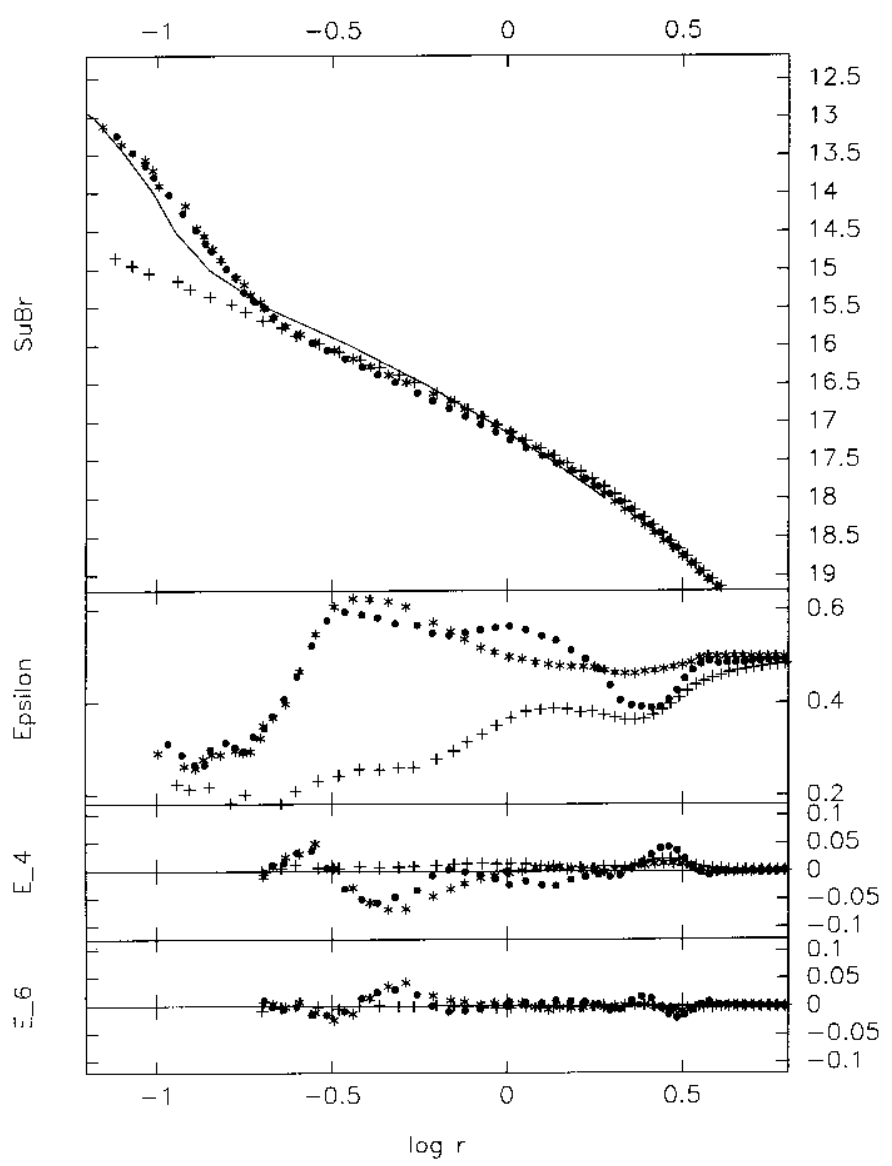

Fig. 8. Results of convolution of a model by the PSF phst0 and deconvolution with PSF's having attenuated wings. The model is the same as in Fig. 7. The abscissae scale is the mean radius $r$ in arcsec and logarithms. Solid line: model $\mathrm{SuBr}$. The crosses refer to the convolved image with phst 0 ; the stars to the deconvolved one with phstx; the dots to the deconvolution by phsty both with 80 iterations. See Fig. 7 for other conventions

right to the nucleus, although its visiblity against the fast increasing bulge light will decrease. Such a disk, smoothly merging with the nucleus, is not the same thing as the inner disks produced by deconvolution in our experiments: in this case a strongly boxy ring surrounds the "nuclear disk". There are cases however, where direct high resolution images show structures somewhat similar to deconvolution artefacts: one exemple is NGC 3115 (see Nieto et al. 1991, Fig. 1). Now, the convolution of an oval galaxy by a roundish PSF will produce disky isophotes in a limited range: this is very clear in our experiments (see Figs. 3 and 7). The technique of Scorza \& Bender (1995) to recover the disk properties in strongly inclined E-S0 galaxies will not be sensitive to this source of errors.

Pending future observations with the "new" HST, one should perhaps remain cautious about part of the published results dealing with the core structure of E-S0 


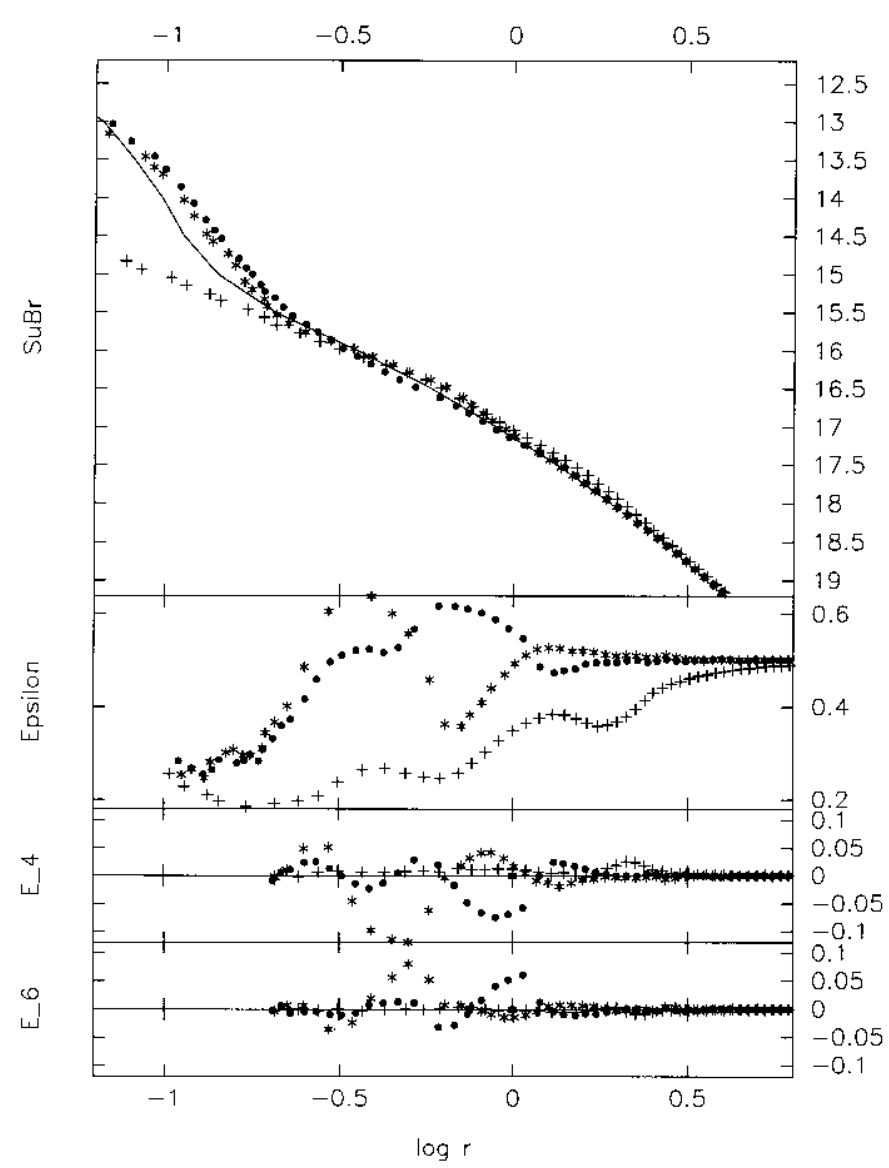

Fig. 9. Results of convolution of a model with phst $1 m$ and deconvolutions with PSF's having a fainter or stronger plateau (phst 1 and phst $1 p$ respectively). The model is the same as in Fig. 7. The abscissae scale is the mean radius $r$ in arcsec and logarithms. Solid line: model SuBr. The crosses refer to the convolved image, the stars to the deconvolved one with phst1, the dots with phst $1 p$, both with 80 iterations. See Figs. 7 or 8 for other conventions

galaxies, and based upon an extensive use of Lucy's deconvolution technique. It should be emphasized that this technique can be useful in various contexts, including the improvement of the light profiles of such cores: it is unwise however to expect that it will recover the correct geometry of elliptical isophotes flatter than E2, unless we have a nice broad core rather than a sharp central component.

Wether or not other deconvolution techniques share the properties here shown for Lucy's method, is a question that will be left to the attention of specialists: various progresses have been made in these techniques, notably in connection with the restoration of HST data. The present author is of the opinion that one cannot be overcautious in the use of deconvolution, when quantitative results are essential. This will remain true for the galaxian cores images from the "new" HST. It will be safer to convolve models with the PSF and compare with the observations... but the deconvolution can help to provide input models for such attempts.

Acknowledgements. J.-L. Nieto shared with me some of his knowledge of the core of galaxies, during a precious collaboration started in 1989 and abruptly ended by his death. His coworker P. Poulain put at my disposal the frames used in the present tests. Part of the calculations for this work were performed with the computing facilities of the Institut d'Astrophysique de Paris.

\section{References}

Burrows C.J., Holtzman J.A., Faber S.M., et al., 1991, ApJ 369, L21

Hodge P.E., Eisenhamer R.A., Shaw R.A., Williamson II R.L., 1993, in Astronomical Data Analysis Software and Systems II, ASP Conf. Ser., 52. In: Hanisch R.J., Brissenden R.J.V., Barnes J. (eds.), 533

Jaffe W., Ford H.C., O'Connell R.W., van den Bosch F.C., Ferrarese L., 1994, AJ 108, 1567

van den Bosch F.C., Ferrarese L., Jaffe W., Ford H.C., O'Connell R.W., 1994, AJ 108, 1579

Ferrarese L., van den Bosch F.C., Ford H.C., Jaffe W., O'Connell R.W, 1994, AJ 108, 1598

Hook R.N., Lucy L.B., 1993, in The Restoration of HST Images and Spectra II, Space Telescope Sci. Inst., 1994. In: Hanisch R.J. and White R.L. (eds.), 86

Krist J., 1993, in Astronomical Data Analysis Software and Systems II, ASP Conf. Ser. 52. In: Hanisch R.J., Brissenden R.J.V., Barnes J. (eds.), 536

Lucy L.B., 1993, in The Restoration of HST Images and Spectra II, Space Telescope Sci. Inst., 1994. In: Hanisch R.J. and White R.L. (eds.), 79

Michard R., Marchal J., 1993, A\&AS 98, 29

Michard R., Marchal J., 1994, A\&AS 105, 481

Mo J., Hanisch R.J., 1993, in The Restoration of HST Images and Spectra II, Space Telescope Sci. Inst., 1994. In: Hanisch R.J. and White R.L. (eds.), 163

Nieto J.L., Bender R., Arnaud J., Surma P., 1991, A\&A 244, L25

Scorza C., Bender R., 1990, A\&A 235, 49

Scorza C., Bender R., 1995, A\&A 293, 20

Simien F., Michard R., 1990, A\&A 227, 11 